\title{
Prognostic significance of lymph node metastasis and lymphadenectomy in early-stage ovarian carcinosarcoma
}

This article was published in the following Dove Press journal: Cancer Management and Research

\author{
Wen-Peng Wang' \\ Ning Li' \\ Yuan-Yuan Zhang' \\ Yu-Tao Gao ${ }^{2}$ \\ Yang-Chun Sun' \\ Li Ge' \\ Ling-Ying $\mathrm{Wu}^{\prime}$
}

'Department of Gynecologic Oncology, National Cancer Center/ National Clinical Research Center for Cancer/Cancer Hospital, Chinese Academy of Medical Sciences and Peking Union Medical College, Beijing, China; ${ }^{2}$ Department of Gynecology, First Affiliated Hospital of Kunming Medical University, Kunming, China

\section{Video abstract}

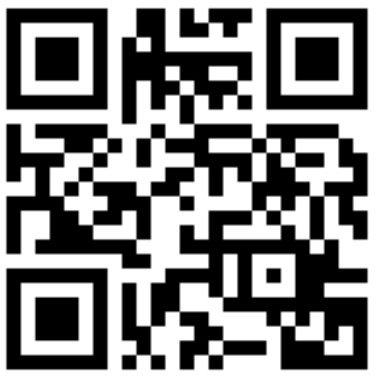

Point your SmartPhone at the code above. If you have a $Q R$ code reader the video abstract will appear. Or use: http://youtu.be/5D0Z3lqxM30

Correspondence: Ling-Ying Wu Department of Gynecologic Oncology, National Cancer Center/National Clinical Research Center for Cancer/Cancer Hospital, Chinese Academy of Medical Sciences and Peking Union Medical College, Panjiayuannanli I7, Chao yang

District, Beijing I0002I, China

Tel +86 I0 87788996

Email wulingying@csco.org.cn
Background: The role that lymph node dissection (LND) plays in the management of ovarian carcinosarcoma (OCS) is unclear due to its rarity. This study investigated lymph node metastasis (LNM) prevalence in women with early OCS and effects of LND and LNM on survival.

Methods: Data of women diagnosed with OCS, whose primary tumor was confined to ovaries (American Joint Committee on Cancer [AJCC] T1) or pelvic cavity (AJCC T2), between 1988 and 2010 were collected from the Surveillance, Epidemiology, and End Results (SEER) database. Patients were classified into lymphadenectomy (LND [+]) and no lymphadenectomy (LND [-]) groups.

Results: A total of 363 women were included. The prevalence of LNM was $9.6 \%$ in AJCC T1 and $16.3 \%$ in AJCC T2. Multivariate analysis showed that LND and AJCC T categories were independent prognostic variables, irrespective of cancer-specific survival (CSS) or overall survival (OS). Subgroup analysis by AJCC T categories revealed that LND (+) group in AJCC T2 had a better survival outcome compared to LND (-) group (CSS, HR [95\% CI] = 0.61 [0.43-0.87]; OS, HR $[95 \% \mathrm{CI}]=0.59[0.42-0.83])$. There was no survival difference between groups in AJCC T1 (CSS, HR [95\% CI] = 0.96 [0.56-1.65]; OS, HR [95\% CI] $=0.88$ [0.56-1.38]). Multivariate analysis was further carried out in LND (+) group and demonstrated that LNM and AJCC T2 had poor CSS and OS. Subgroup analysis by AJCC T categories showed that worse survival was observed in LNM (+) group compared to LNM (-) group in AJCC T2 (CSS, HR [95\% CI] $=3.62$ [1.50-8.73]; OS, HR [95\% CI] = 3.71 [1.59-8.68]) but not in AJCC T1 (CSS, HR [95\% $\mathrm{CI}]=1.78[0.50-6.37] ;$ OS, HR $[95 \% \mathrm{CI}]=1.97$ [0.61-6.39]).

Conclusion: Regional lymphadenectomy should be performed in patients with AJCC T2 OCS. LND and LNM were not significantly associated with prognosis in AJCC T1 while LNM had a trend toward worse survival.

Keywords: ovarian mesodermal mixed tumor, ovarian müllerian mixed tumor, lymph node examined, lymphatic metastasis

\section{Introduction}

Ovarian carcinosarcoma (OCS), also known as müllerian mixed tumor or mesodermal mixed tumor, is a rare but aggressive malignancy histologically composed of carcinomatous and sarcomatous elements. ${ }^{1}$ Previous studies have shown that an older age at onset, higher International Federation of Gynecology and Obstetrics (FIGO) stage, and worse prognosis are observed in women with OCS when compared to women with epithelial ovarian carcinoma (EOC)., ${ }^{2,3}$ Given the rarity of OCS, it is difficult to carry out prospective clinical trials with this disease. Therefore, the mainstay of treatment for OCS is primarily based on management experience of EOC and data from retrospective 
studies including a small number of cases. ${ }^{4-9}$ To date there is no uniform agreement about the optimal management of OCS. Previous series of studies have observed that there is survival benefit for women with OCS who undergo cytoreductive surgery followed by platinum-based chemotherapy. ${ }^{4-9}$ Therefore, debulking surgery combined with chemotherapy is the preferred treatment for OCS.

The Surveillance, Epidemiology, and End Results (SEER) database, covering approximately $28 \%$ of the population of the USA, collects data on cancer cases from different districts and sources. Rauh-Hain et al summarized OCS data in the SEER database and found that OCS women with lymph node dissection (LND) had a lower lymph node metastasis (LNM) rate than women with high grade serous ovarian carcinoma (HGSOC) women with LND. ${ }^{2}$ Garg et al compared OCS survival rates with those of uterine carcinosarcoma (UCS) by analyzing the SEER database and showed that LND was significantly associated with an improved OCS and UCS survival. ${ }^{10}$ However, a multi-center study by the Taiwanese Gynecologic Oncology Group found that pelvic LNM for carcinosarcoma of the ovary, fallopian tube, and peritoneum trended toward worse survival without statistical significance. ${ }^{11}$ The condition of LNM in early OCS, its relationship with prognosis, and whether LND is necessary in early OCS have not been previously reported. The purpose of this study is to explore the prognostic effect of lymphatic metastasis and resection in early OCS based on a large sample from SEER.

\section{Patients and methods Patients}

OCS data were collected from the SEER database, consisting of 18 population-based cancer registries. Clinicopathological information was extracted using the "case listing" option of the SEER*Stat 8.3.4 software. Preliminary selection criteria for study cases included: 1) diagnosis of OCS or müllerian mixed tumor or mesodermal mixed tumor; 2) histological confirmation of disease; 3) diagnosis between 1988 and 2010. A total of 2,275 patients with OCS met our inclusion criteria. Exclusion criteria were: 1) multiple primary malignancies; 2) non-American Joint Committee on Cancer (AJCC) T1 and T2; 3) distant metastasis; 4) unknown regional lymph nodes examination; 5) unknown survival time; 6) no surgery performed. Finally, 363 patients with early OCS were identified (Figure 1). Institutional review board approval was obtained from the National Cancer Center/Cancer Hospital, Chinese Academy of Medical Science and Peking Union Medical College (Beijing, China). Cancer is a reportable disease under the laws of all 50 states in the US and informed consent is not required for data released by the SEER database. SEER data are de-identified before release and do not contain any personally identifiable information.

\section{AJCC TNM (primary tumor, regional lymph node, and distant metastasis) staging}

OCS staging between 1988 and 2003 in SEER database was defined by AJCC TNM categories 3rd edition. SEER Program Comparative Staging Guide for Cancer describes AJCC TNM categories 3rd edition OCS definition in detail (https://seer.cancer. gov/archive/manuals/historic/comp stage1.1.pdf). OCS staging between 2004 and 2009 was derived from AJCC TNM categories 6 th edition. OCS staging in 2010 was determined by AJCC TNM categories 7th edition. The description of AJCC TNM categories 6 th and 7 th edition of the ovary refers to Collaborative Stage Data Collection System. The definition of AJCC T1 and T2 categories $3 \mathrm{rd}$ edition is the same as 6 th and 7 th edition.

AJCC T1: tumor limited to one or both ovaries; AJCC T2: tumor involves one or both ovaries with pelvic extension; AJCC N0: no regional LNM; AJCC N1: regional LNM; AJCC M0: no distant metastasis; AJCC M1: distant metastasis; AJCC T1N0M0: FIGO stage I; AJCC T1aN0M0, T1bN0M0, T1cN0M0: FIGO Ia, Ib, Ic; AJCC T2N0M0: FIGO II; AJCC T2aN0M0, T2bN0M0, T2cN0M0: FIGO stage IIa, IIb, IIc; AJCC T1/2N1M0: FIGO IIIa.

\section{Statistical analysis}

Frequency distribution of clinicopathological variables between LND (+) and LND (-) groups was compared with the $\chi^{2}$ test and the Fisher exact test. The Mann-Whitney test was used to compare the mean age of the two groups. The LNM rate of different AJCC T categories was calculated as the number of patients with LNM divided by patients with lymphadenectomy in each AJCC T category. Survival curves were generated using the Kaplan-Meier method. Univariate and multivariate analyses of the effects of categorical variables on the survival were performed using Cox proportional hazards model. Multivariate Cox regression analysis was used to identify independent prognostic factors for all cases $(n=363)$ and cases who underwent lymphadenectomy $(n=186)$. The condition of LNM was considered unknown for patients who did not undergo lymphadenectomy. Therefore, LNM was only included in multivariate Cox regression analysis of cases who underwent lymphadenectomy $(\mathrm{N}=186)$. We used IBM SPSS 22.0 and Graph Pad Prism 5.0 to analyze data. A $p<0.05$ was considered statistically significant. 


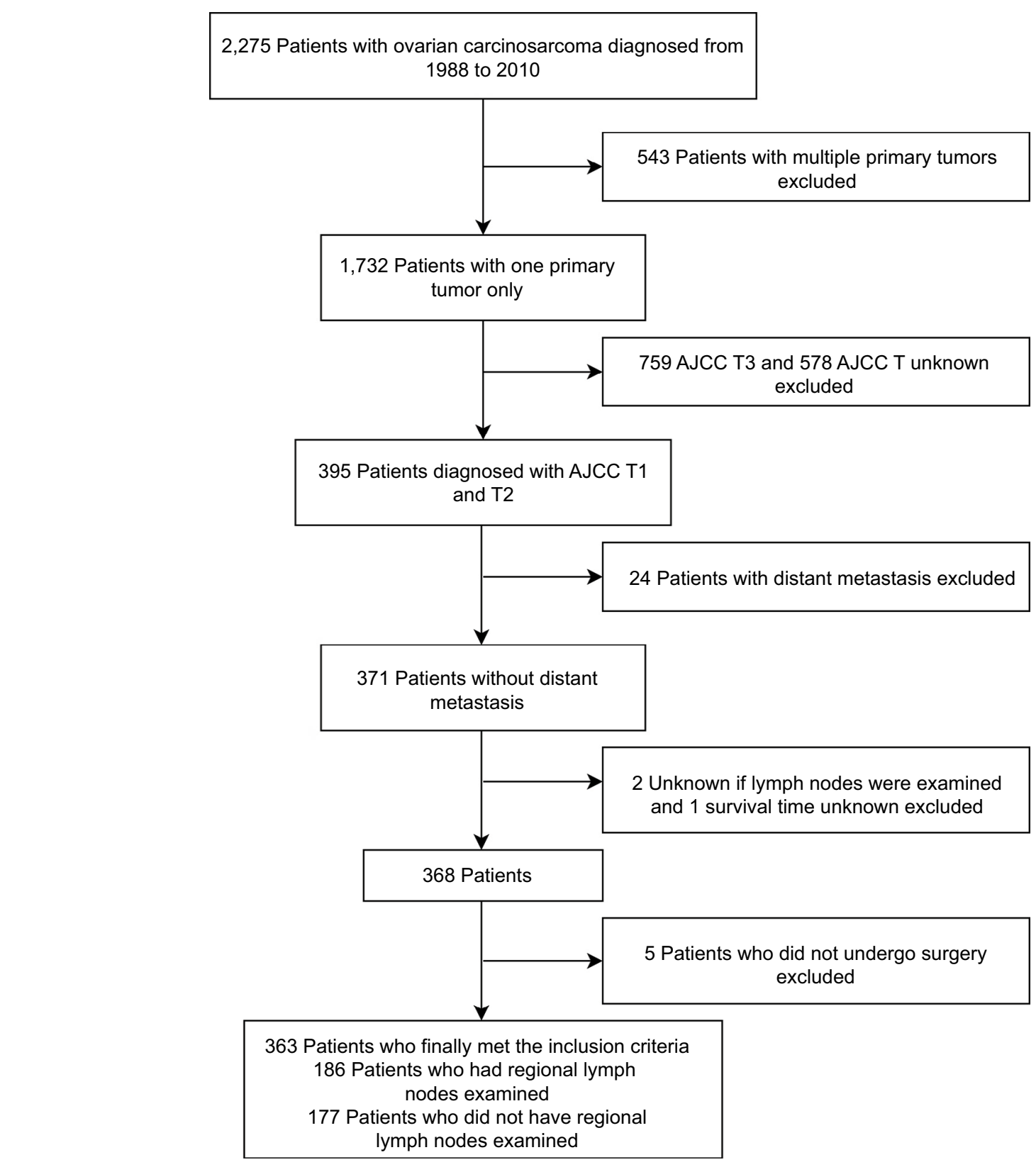

Figure I Screening flow chart for ovarian carcinosarcoma.

Abbreviations: AJCC, American Joint Committee on Cancer; T, primary tumor.

\section{Results}

A cohort of 363 patients with OCS met the study eligibility criteria. These patients were divided into groups, 186 patients who underwent lymphadenectomy (LND [+] group) and 177 patients who did not undergo lymphadenectomy (LND [-] group). Table 1 shows the demographic and clinicopathological features of both groups. LND (+) group had a younger mean age compared to LND (-) group ( 60.52 years vs. 65.43 years, $p<0.001)$. A higher proportion of patients with OCS diagnosed between 2001 and 2010 underwent regional lymphadenectomy (LND [+] $62.4 \%$ vs. LND [-]
44.6\%) when compared to those diagnosed between 1988 and $2000(\mathrm{LND}[+] 37.6 \%$ vs. LND [-] 55.4\%, $p=0.001)$. In LND (+) group, the LNM rate of patients with AJCC T1 was 9.6\%. Contrastingly, 16.3\% patients had LNM in AJCC T2 (Table 2).

Multivariate Cox regression analysis in patients with OCS showed that LND $(p=0.042)$ and early AJCC T categories $(p<0.001)$ were positively associated with cancer-specific survival (CSS) after adjusting for age, registry district, tumor laterality, AJCC T categories, and LND. These adjusted variables had statistically significant differences in univariate 
Table I Demographic and clinicopathological characteristics of patients with OCS stratified by LND

\begin{tabular}{|c|c|c|c|}
\hline Variables & LND (+) & LND (-) & $p$-value \\
\hline Number & 186 (5I.24\%) & I 77 (48.76\%) & \\
\hline \multicolumn{4}{|l|}{ Age at diagnosis } \\
\hline Mean age (SD) & $60.52(13.15)$ & $65.43(15.11)$ & $<0.001$ \\
\hline Race & & & 0.922 \\
\hline White & I58 (84.9\%) & I5I (85.3\%) & \\
\hline Non-white & $28(15.1 \%)$ & $26(14.7 \%)$ & \\
\hline SEER registry, \% & & & 0.128 \\
\hline Central & 47 (25.3\%) & $5 \mathrm{I}(28.8 \%)$ & \\
\hline Eastern & $43(23.1 \%)$ & 53 (29.9\%) & \\
\hline Western & $96(51.6 \%)$ & 73 (4I.2\%) & \\
\hline Date range & & & 0.001 \\
\hline $1988-2000$ & $70(37.6 \%)$ & 98 (55.4\%) & \\
\hline $200 I-2010$ & $116(62.4 \%)$ & 79 (44.6\%) & \\
\hline Tumor laterality & & & 0.073 \\
\hline Unilateral & I 48 (79.6\%) & 140 (79.1\%) & \\
\hline Bilateral & 37 (19.9\%) & $30(16.9 \%)$ & \\
\hline Paired* & I (0.5\%) & 7 (4.0\%) & \\
\hline AJCC T categories & & & 0.668 \\
\hline Tla & $56(30.1 \%)$ & $4 \mathrm{I}(23.2 \%)$ & \\
\hline TIb & $6(3.2 \%)$ & $5(2.8 \%)$ & \\
\hline TIc & 28 (I5.1\%) & 21 (11.9\%) & \\
\hline TI NOS & $4(2.2 \%)$ & $7(4.0 \%)$ & \\
\hline $\mathrm{T} 2 \mathrm{a}$ & $16(8.6 \%)$ & $16(9.0 \%)$ & \\
\hline $\mathrm{T} 2 \mathrm{~b}$ & $36(19.4 \%)$ & $43(24.3 \%)$ & \\
\hline $\mathrm{T} 2 \mathrm{c}$ & $33(17.7 \%)$ & 35 (19.8\%) & \\
\hline T2 NOS & 7 (3.8\%) & $9(5.1 \%)$ & \\
\hline Radiation & & & 0.395 \\
\hline Yes & 7 (3.8\%) & $10(5.6 \%)$ & \\
\hline No & $179(96.2 \%)$ & $167(94.4 \%)$ & \\
\hline
\end{tabular}

Note: *No information concerning laterality.

Abbreviations: OCS, ovarian carcinosarcoma; LND, lymph node dissection; SEER, Surveillance, Epidemiology, and End Results; AJCC, American Joint Committee on Cancer; T, primary tumor; NOS, not otherwise specified.

analysis of CSS. Lymphadenectomy $(p=0.031)$, early AJCC T categories $(p<0.001)$, and age $\leq 63(p<0.001)$ were associated with an improved overall survival (OS) in multivariate analysis after adjusting for age, registry district, AJCC T categories, and LND that influenced OS in univariate analysis (Table 3).

In Kaplan-Meier survival analysis stratified by AJCC $\mathrm{T}$ categories, we found that a higher survival benefit was observed in LND (+) group than LND (-) group in AJCC $\mathrm{T} 2$ ( $p=0.006$ for CSS, $p=0.002$ for OS; Figure 2C and D), but there was no statistically significant difference between LND (+) group and LND (-) group in AJCC T1 $(p=0.887$ for CSS, $p=0.582$ for OS; Figure 2A and B).

Multivariate analysis of LND $(+)$ group showed that LNM ( $p=0.001$ for CSS, $p=0.004$ for OS) and AJCC T categories ( $p<0.001$ for CSS, $p=0.002$ for OS) were independent prognostic factors of patients. Older age was negatively
Table 2 LNM rate of OCS in different AJCC T categories

\begin{tabular}{llll}
\hline $\begin{array}{l}\text { AJCC T } \\
\text { categories }\end{array}$ & $\begin{array}{l}\text { Patients } \\
\text { with LND }\end{array}$ & $\begin{array}{l}\text { Patients } \\
\text { with LNM }\end{array}$ & Rate \\
\hline TI & 94 & 9 & \\
TIa & 56 & 5 & $9.6 \%$ \\
TIb & 6 & 0 & $8.9 \%$ \\
TIc & 28 & 4 & $0.0 \%$ \\
TI NOS & 4 & 0 & $14.3 \%$ \\
T2 & 92 & 15 & $0.0 \%$ \\
T2a & 16 & 4 & $16.3 \%$ \\
T2b & 36 & 7 & $25.0 \%$ \\
T2c & 33 & 3 & $19.4 \%$ \\
T2 NOS & 7 & 1 & $9.1 \%$ \\
\hline
\end{tabular}

Abbreviations: LNM, lymph node metastasis; OCS, ovarian carcinosarcoma; AJCC, American Joint Committee on Cancer; T, primary tumor; LND, lymph node dissection; NOS, not otherwise specified.

associated with OS ( $p=0.042)$; it was not confirmed to be related with CSS ( $p=0.187$; Table 4$)$.

In LND (+) group, the subgroup analysis by AJCC T categories revealed that patients with LNM had lower CSS $(p=0.004$; Figure $3 \mathrm{C})$ and $\mathrm{OS}(p=0.003$; Figure 3D) rate than patients without LNM in AJCC T2. No survival difference was found between LNM (+) group and LNM (-) group in AJCC T1 ( $p=0.377$ for CSS, $p=0.257$ for OS; Figure $3 \mathrm{~A}$ and $\mathrm{B}$ ).

Figure $4 \mathrm{~A}$ and $\mathrm{B}$ shows the survival differences of various AJCC T categories combined with various AJCC $\mathrm{N}$ categories in LND (+) group. Any two comparisons of four groups by Log-rank method showed that patients with AJCC T2N1M0 had a poorer survival outcome than patients with AJCC T2N0M0 ( $p=0.006$ for CSS, $p=0.005$ for OS), T1N1M0 ( $p=0.034$ for CSS, $p=0.043$ for OS) and T1N0M0 $(p<0.001$ for CSS, $p<0.001$ for OS). However, there was no statistically significant survival difference between patients with AJCC T1N1M0 and patients with AJCC T1N0M0 ( $p=$ 0.377 for CSS, $p=0.257$ for OS; Table S1).

\section{Discussion}

OCS consists of malignant epithelial and stromal components that accounts for $1 \%$ to $4 \%$ of all EOC. ${ }^{1,12}$ Previous series of studies have noted that OCS with poor survival outcome has more aggressive tumor biology compared to other histological subsets of EOC. ${ }^{1,13,14}$ Considering the rarity of OCS, publications on the role that LND plays in the management of OCS are few. Because of this, making full use of large sample databases such as SEER and the National Cancer Database can enable powerful analysis of clinicopathological characteristics and survival outcomes from a greater number of women with OCS. 
Table 3 Multivariate analysis for significant prognostic factors in patients with OCS $(n=363)$

\begin{tabular}{|c|c|c|c|c|c|c|c|c|c|}
\hline \multirow[t]{3}{*}{ Variables } & \multirow[t]{3}{*}{$\mathbf{n}^{\#}$} & \multicolumn{4}{|l|}{ CSS } & \multicolumn{4}{|l|}{ OS } \\
\hline & & \multicolumn{2}{|c|}{ Univariate analysis } & \multicolumn{2}{|c|}{ Multivariate analysis } & \multicolumn{2}{|c|}{ Univariate analysis } & \multicolumn{2}{|c|}{ Multivariate analysis } \\
\hline & & HR (95\% Cl) & $p$-value & HR (95\% Cl) & $p$-value & HR (95\% Cl) & $p$-value & HR (95\% Cl) & $\overline{p \text {-value }}$ \\
\hline LND & & & 0.009 & & 0.042 & & 0.003 & & 0.031 \\
\hline$(+)$ & 186 & Reference & & Reference & & Reference & & Reference & \\
\hline$(-)$ & 177 & $1.46(1.10-1.94)$ & & $1.36(1.01-1.84)$ & & $1.49(1.15-1.93)$ & & $1.34(1.03-1.76)$ & \\
\hline AJCC T categories & & & $<0.001$ & & $<0.001$ & & $<0.001$ & & $<0.001$ \\
\hline $\mathrm{Tla}$ & 97 & Reference & & Reference & & Reference & & Reference & \\
\hline TIb & II & $1.54(0.54-4.40)$ & & $1.55(0.52-4.62)$ & & $0.98(0.35-2.73)$ & & $0.98(0.35-2.73)$ & \\
\hline TIC & 49 & $2.15(1.22-3.79)$ & & $2.14(1.21-3.78)$ & & $1.84(1.14-2.98)$ & & $1.86(1.15-3.01)$ & \\
\hline TI NOS & 11 & $1.37(0.48-3.92)$ & & $1.43(0.49-4.13)$ & & $1.05(0.42-2.66)$ & & $1.20(0.47-3.05)$ & \\
\hline $\mathrm{T} 2 \mathrm{a}$ & 32 & $3.06(1.67-5.60)$ & & $2.76(1.48-5.13)$ & & $2.47(1.45-4.18)$ & & $2.07(1.21-3.55)$ & \\
\hline $\mathrm{T} 2 \mathrm{~b}$ & 79 & $3.5 I(2.18-5.67)$ & & $3.30(2.00-5.45)$ & & $2.81(1.87-4.20)$ & & $2.58(I .7 I-3.89)$ & \\
\hline $\mathrm{T} 2 \mathrm{c}$ & 68 & $4.62(2.87-7.42)$ & & $4.15(2.52-6.83)$ & & $3.52(2.34-5.29)$ & & $2.97(1.96-4.50)$ & \\
\hline T2 NOS & 16 & 5.88 (3.09-11.17) & & $5.43(2.84-10.39)$ & & $4.66(2.59-8.37)$ & & $4.09(2.27-7.38)$ & \\
\hline Age (years) & & & $<0.001$ & & 0.053 & & $<0.001$ & & $<0.001$ \\
\hline$\leq 63$ & 175 & Reference & & Reference & & Reference & & Reference & \\
\hline$>63$ & 188 & $1.72(1.29-2.29)$ & & $1.36(1.00-1.85)$ & & $2.19(1.67-2.87)$ & & I.83 (1.37-2.44) & \\
\hline SEER registry, \% & & & 0.019 & & 0.064 & & 0.036 & & 0.089 \\
\hline Central & 98 & Reference & & Reference & & Reference & & Reference & \\
\hline Eastern & 96 & $1.47(1.01-2.13)$ & & $1.34(0.92-1.96)$ & & $1.41(1.00-1.99)$ & & $1.36(0.96-1.94)$ & \\
\hline Western & 169 & $0.93(0.65-1.33)$ & & $0.90(0.63-1.30)$ & & $0.96(0.70-1.32)$ & & $0.99(0.7|-| .38)$ & \\
\hline Tumor laterality & & & 0.018 & & 0.939 & & 0.103 & & \\
\hline Unilateral & 288 & Reference & & Reference & & Reference & & & \\
\hline Bilateral & 67 & $1.49(1.07-2.09)$ & & $1.04(0.72-1.50)$ & & I.27 (0.92-I.75) & & & \\
\hline Paired* & 8 & $2.14(0.95-4.85)$ & & $1.14(0.49-2.66)$ & & $1.93(0.91-4.11)$ & & & \\
\hline Race & & & 0.701 & & & & 0.893 & & \\
\hline White & 309 & Reference & & & & Reference & & & \\
\hline Non-white & 54 & $0.92(0.62-1.39)$ & & & & $0.98(0.68-1.40)$ & & & \\
\hline Date range & & & 0.888 & & & & 0.623 & & \\
\hline $1988-2000$ & 168 & Reference & & & & Reference & & & \\
\hline $2001-2010$ & 195 & $1.02(0.77-1.36)$ & & & & $0.94(0.72-1.22)$ & & & \\
\hline Radiation & & & 0.189 & & & & 0.064 & & \\
\hline Yes & 17 & Reference & & & & Reference & & & \\
\hline No & 346 & $0.66(0.36-1.22)$ & & & & $0.60(0.35-1.03)$ & & & \\
\hline
\end{tabular}

Note: \#Number of patients; *no information concerning laterality.

Abbreviations: OCS, ovarian carcinosarcoma; CSS, cancer-specific survival; OS, overall survival; LND, lymph node dissection; AJCC, American Joint Committee on Cancer; T, primary tumor; NOS, not otherwise specified; SEER, Surveillance, Epidemiology, and End Results.

Garg et al used the SEER database to make a comparison of prognostic factors between 924 women with OCS and 2,759 women with UCS and found that LND significantly improved survival outcomes of OCS and UCS. ${ }^{10}$ Rauh-Hain et al analyzed 14,753 women diagnosed between 1998 and 2009 from the SEER database and demonstrated a lower LNM rate in women with OCS when compared to women with HGSOC (35.4\% vs. 54.7\%, $p<0.001) .{ }^{2}$ In an analysis of 47 cases with primary OCS, six women (35.3\%) had positive lymph nodes among 17 women who underwent LND. ${ }^{15}$ Our study used the SEER database to show the prevalence of LNM, and the relationship between survival outcomes and LND as well as LNM when OCS was limited to ovaries (AJCC T1) or confined to the pelvic cavity (AJCC T2).
Complete surgical cytoreduction is significantly associated with improved survival according to small retrospective studies. ${ }^{4,6,16}$ It is well-known that National Comprehensive Cancer Network guidelines for EOC recommend comprehensive surgical staging be performed in early EOC. However, whether regional lymphadenectomy should be performed in early OCS deserves further investigation. Women with OCS tend to present at an older age when compared to women with EOC. ${ }^{2,3,8}$ In our study, the mean age at diagnosis of LND (+) group (60.52 years old) was younger than LND (-) group (65.43 years old). This indirectly suggests that surgeons may take a patient's age into consideration when deciding the scope of debulking surgery. Additionally, we found that more patients with OCS between 2001 and 2010 received 
A
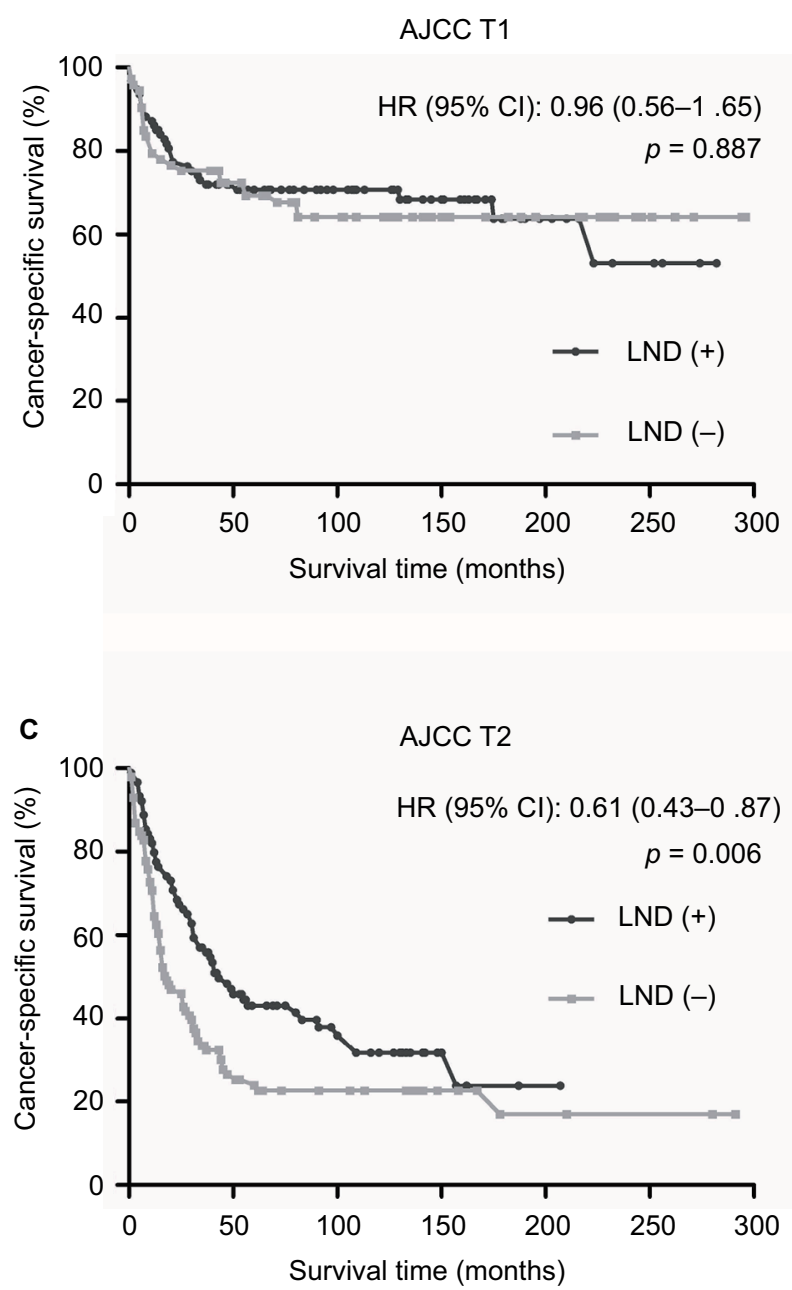

B

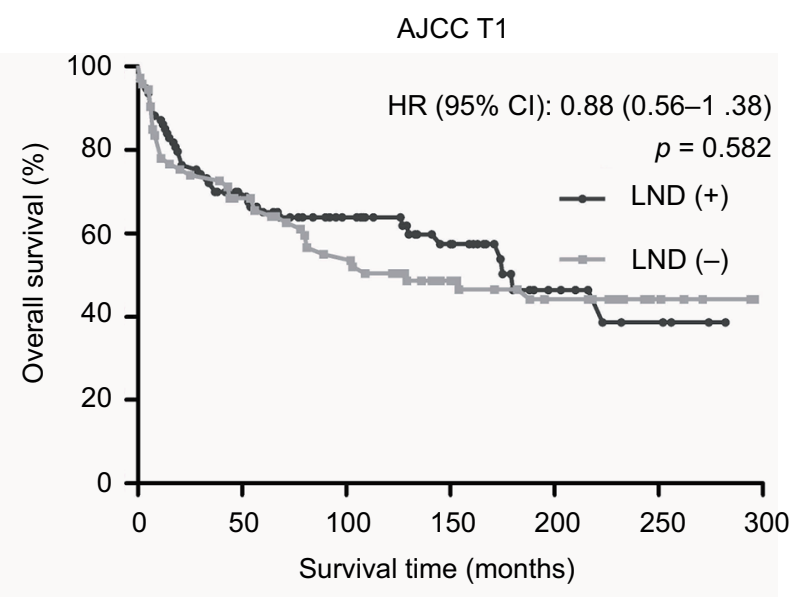

D

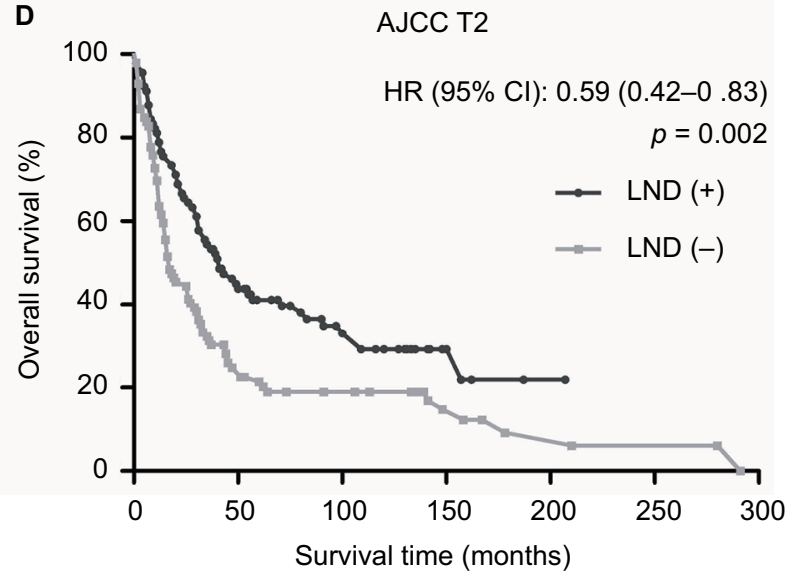

Figure 2 Kaplan-Meier survival curves according to LND status following stratification by AJCC T categories.

Notes: (A) Cancer-specific survival for AJCC TI. (B) Overall survival for AJCC TI. (C) Cancer-specific survival for AJCC T2. (D) Overall survival for AJCC T2.

Abbreviations: LND, lymph node dissection; AJCC, American Joint Committee on Cancer; T, primary tumor.

regional lymphadenectomy than those between 1988 and 2000. Rauh-Hain et al made a treatment comparison between OCS and HGSOC patients stratified by period of diagnosis and showed that the proportion of lymphadenectomy in patients with OCS increased from 2003 to 2011 (2003-2005 $47.9 \%$ vs. $2006-200848.6 \%$ vs. $2009-201153.0 \%) .{ }^{3}$ Our study showed that the LNM rate of OCS was $9.6 \%$ in AJCC T1 and $16.3 \%$ in AJCC T2. Research on early OCS is few owing to its rarity. To our knowledge, there is no literature reporting on LNM in early OCS. We made a comparison between OCS and UCS in terms of LNM prevalence. A previous study demonstrated that $16.7 \%$ (34/203) of patients with early UCS had retroperitoneal LNM; it was similar to the LNM prevalence of OCS in AJCC T2. ${ }^{17}$

Jernigan et al showed that age, stage, and complete surgical cytoreduction were independent prognostic variables in a study of 47 women with $\mathrm{OCS}^{6}$ In our study, multivariate analysis identified an independent positive association of early AJCC T categories and LND with CSS and OS. Older age was negatively associated with OS but not CSS in multivariate analysis. Younger age, early diagnosis, early stage, surgical treatment, and LND were reported to be positively associated with survival outcomes in $\mathrm{OCS}^{2}$ We made a survival comparison between LND (+) and LND (-) groups following stratification by AJCC T categories. In subgroup analysis, no survival difference was observed between LND $(+)$ and LND (-) groups in AJCC T1 (CSS, HR = 0.96, $95 \% \mathrm{CI}=0.56-1.65$; OS, $\mathrm{HR}=0.88,95 \% \mathrm{CI}=0.56-1.38)$. LND significantly improved the survival of patients with AJCC T2 $(\mathrm{CSS}, \mathrm{HR}=0.61,95 \% \mathrm{CI}=0.43-0.87$; OS, HR = $0.59,95 \% \mathrm{CI}=0.42-0.83$ ).

In order to further analyze the relationship of LNM and the survival in early OCS, we performed multivariate Cox regression analysis with the LND (+) group. LNM and 
Table 4 Multivariate analysis of factors associated with the survival of patients with OCS who underwent LND $(n=186)$

\begin{tabular}{|c|c|c|c|c|c|c|c|c|c|}
\hline \multirow[t]{3}{*}{ Variables } & \multirow[t]{3}{*}{$\mathbf{n}^{\#}$} & \multicolumn{4}{|l|}{ CSS } & \multicolumn{4}{|l|}{ OS } \\
\hline & & \multicolumn{2}{|c|}{ Univariate analysis } & \multicolumn{2}{|c|}{ Multivariate analysis } & \multicolumn{2}{|c|}{ Univariate analysis } & \multicolumn{2}{|c|}{ Multivariate analysis } \\
\hline & & HR (95\% Cl) & p-value & HR (95\% Cl) & p-value & HR (95\% Cl) & $p$-value & HR (95\% Cl) & $p$-value \\
\hline LNM & & & 0.006 & & 0.001 & & 0.004 & & 0.004 \\
\hline$(+)$ & 24 & Reference & & Reference & & Reference & & Reference & \\
\hline$(-)$ & 162 & $0.46(0.27-0.80)$ & & $0.38(0.2 \mathrm{I}-0.68)$ & & $0.47(0.28-0.78)$ & & $0.44(0.25-0.77)$ & \\
\hline AJCC T categories & & & 0.001 & & $<0.001$ & & 0.004 & & 0.002 \\
\hline Tla & 56 & Reference & & Reference & & Reference & & Reference & \\
\hline TIb & 6 & $1.14(0.26-5.00)$ & & $1.25(0.29-5.48)$ & & $0.74(0.17-3.13)$ & & $0.65(0.15-2.82)$ & \\
\hline TIc & 28 & $1.44(0.65-3.17)$ & & $1.25(0.56-2.78)$ & & $1.17(0.59-2.35)$ & & $1.17(0.58-2.37)$ & \\
\hline TI NOS & 4 & $1.63(0.37-7.20)$ & & $1.77(0.40-7.84)$ & & $1.01(0.24-4.34)$ & & $1.27(0.29-5.52)$ & \\
\hline $\mathrm{T} 2 \mathrm{a}$ & 16 & $1.86(0.76-4.53)$ & & $1.44(0.58-3.59)$ & & $1.43(0.64-3.20)$ & & $1.02(0.45-2.33)$ & \\
\hline $\mathrm{T} 2 \mathrm{~b}$ & 36 & $2.23(1.12-4.44)$ & & $2.18(1.09-4.35)$ & & $1.86(1.02-3.39)$ & & $1.76(0.95-3.24)$ & \\
\hline $\mathrm{T} 2 \mathrm{c}$ & 33 & $4.26(2.27-7.98)$ & & $4.62(2.46-8.69)$ & & $3.03(1.73-5.31)$ & & $3.06(I .73-5.4 I)$ & \\
\hline T2 NOS & 7 & $3.82(1.48-9.87)$ & & $3.32(1.28-8.61)$ & & $3.19(1.36-7.50)$ & & $3.04(1.29-7.18)$ & \\
\hline Age (years) & & & 0.187 & & & & 0.010 & & 0.042 \\
\hline$\leq 63$ & 106 & Reference & & & & Reference & & Reference & \\
\hline$>63$ & 80 & $1.33(0.87-2.03)$ & & & & $1.67(1.13-2.46)$ & & $1.54(1.02-2.34)$ & \\
\hline SEER registry, \% & & & 0.357 & & & & $0.56 \mathrm{I}$ & & \\
\hline Central & 47 & Reference & & & & Reference & & & \\
\hline Eastern & 43 & $1.38(0.77-2.46)$ & & & & $1.27(0.74-2.19)$ & & & \\
\hline Western & 96 & $0.97(0.57-1.64)$ & & & & $0.99(0.62-1.61)$ & & & \\
\hline Tumor laterality & & & 0.457 & & & & 0.837 & & \\
\hline Unilateral & 148 & Reference & & & & Reference & & & \\
\hline Bilateral & 37 & $1.21(0.73-2.00)$ & & & & $1.05(0.65-1.70)$ & & & \\
\hline Paired* & 1 & - & & & & - & & & \\
\hline Race & & & 0.776 & & & & 0.957 & & \\
\hline White & 158 & Reference & & & & Reference & & & \\
\hline Non-white & 28 & $0.92(0.50-1.69)$ & & & & $0.99(0.57-I .7 I)$ & & & \\
\hline Date range & & & 0.976 & & & & 0.492 & & \\
\hline $1988-2000$ & 70 & Reference & & & & Reference & & & \\
\hline $200 I-2010$ & 116 & $0.99(0.64-1.55)$ & & & & $0.87(0.58-1.30)$ & & & \\
\hline Radiation & & & 0.225 & & & & 0.025 & & 0.177 \\
\hline Yes & 7 & Reference & & & & Reference & & Reference & \\
\hline No & 179 & $0.54(0.20-1.47)$ & & & & $0.39(0.17-0.89)$ & & $0.54(0.22-1.32)$ & \\
\hline
\end{tabular}

Notes: "Number of patients; *no information concerning laterality; one case with "paired" was not included in univariate analysis.

Abbreviations: OCS, ovarian carcinosarcoma; CSS, cancer-specific survival; OS, overall survival; LND, lymph node dissection; LNM, lymph node metastasis; AJCC, American Joint Committee on Cancer; T, primary tumor; NOS, not otherwise specified; SEER, Surveillance, Epidemiology, and End Results.

advanced AJCC T categories were significantly associated with a worse CSS and OS. Gynecologic Cancer InterGroup provided a consensus review for OCS and UCS. Women with OCS and UCS have a worse survival rate with a high risk of recurrence compared to those with endometrioid or highgrade serous histologies. Complete surgical staging combined with chemotherapy is recommended for patients with OCS or UCS regardless of early or advanced stage disease. ${ }^{18}$ To date no research has clearly shown the relationship between LNM and the prognosis of OCS. However, a study published by Şükür et al. demonstrated that LNM was the most important predictor of UCS survival in a multivariate analysis. ${ }^{19}$ The Cox model identified an independent negative association between older age and OS but not CSS, which suggests that the elderly with OCS may die of other diseases or causes rather than OCS. Subgroup analysis revealed that LNM had an impact on CSS and OS in AJCC T2 (CSS, HR = 3.62, $95 \% \mathrm{CI}=1.50-8.73 ; \mathrm{OS}, \mathrm{HR}=3.71,95 \% \mathrm{CI}=1.59-8.68$ ) but not AJCC T1 $(\mathrm{CSS}, \mathrm{HR}=1.78,95 \% \mathrm{CI}=0.50-6.37$; OS, $\mathrm{HR}=1.97,95 \% \mathrm{CI}=0.61-6.39)$.

As we all know, gynecologic oncologists define tumor staging by FIGO staging. Because of the rarity of OCS cases, OCS staging mainly refers to epithelial ovarian cancer FIGO staging. At presentation, regional LNM in early OCS is classified as FIGO stage IIIa, but we found that patients with AJCC T1N1M0 had a better prognosis when compared to patients with AJCC T2N1M0. Meanwhile, there was no significant difference seen between the survival of patients with AJCC T1N1M0 and AJCC T1N0M0, while a worse survival trend was observed in AJCC T1N1M0 when compared to AJCC 
A

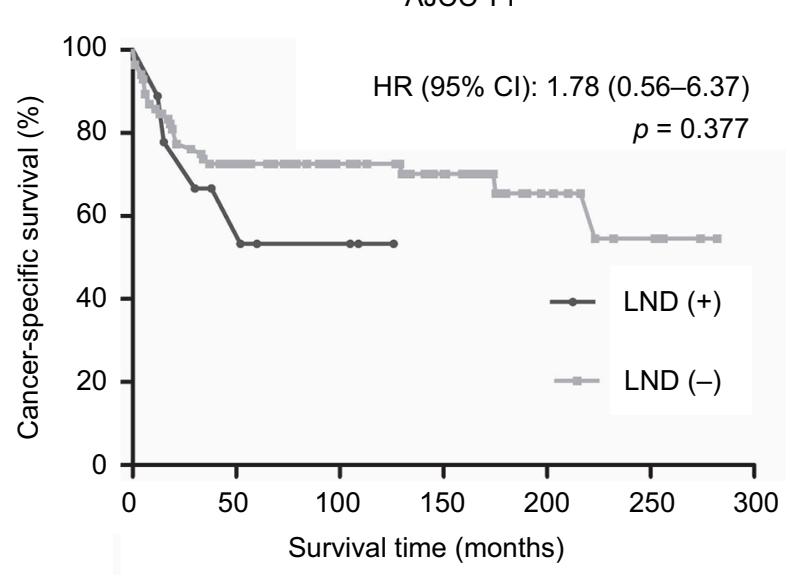

C

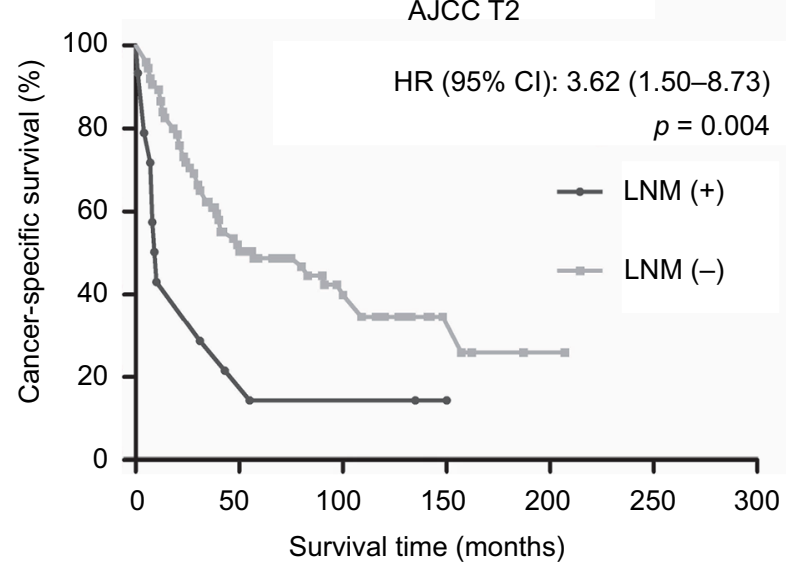

B

AJCC T1

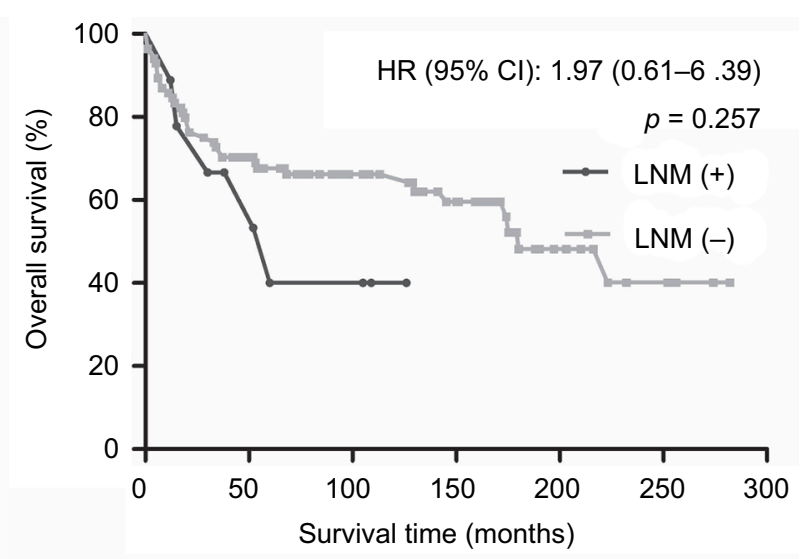

D

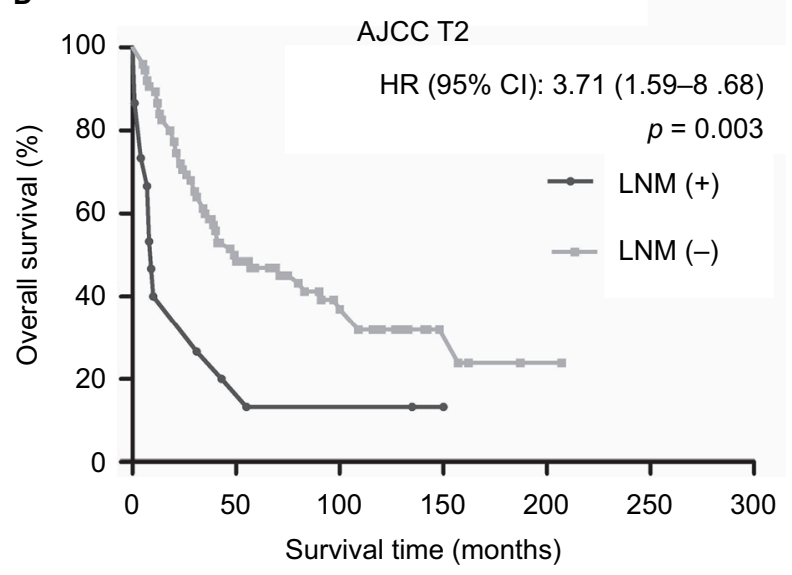

Figure 3 Kaplan-Meier survival curves according to LNM status following stratification by AJCC T categories in LND (+) group.

Notes: (A) Cancer-specific survival for AJCC TI. (B) Overall survival for AJCC TI. (C) Cancer-specific survival for AJCC T2. (D) Overall survival for AJCC T2. Abbreviations: LNM, lymph node metastasis; LND, lymph node dissection; AJCC, American Joint Committee on Cancer; T, primary tumor.

A
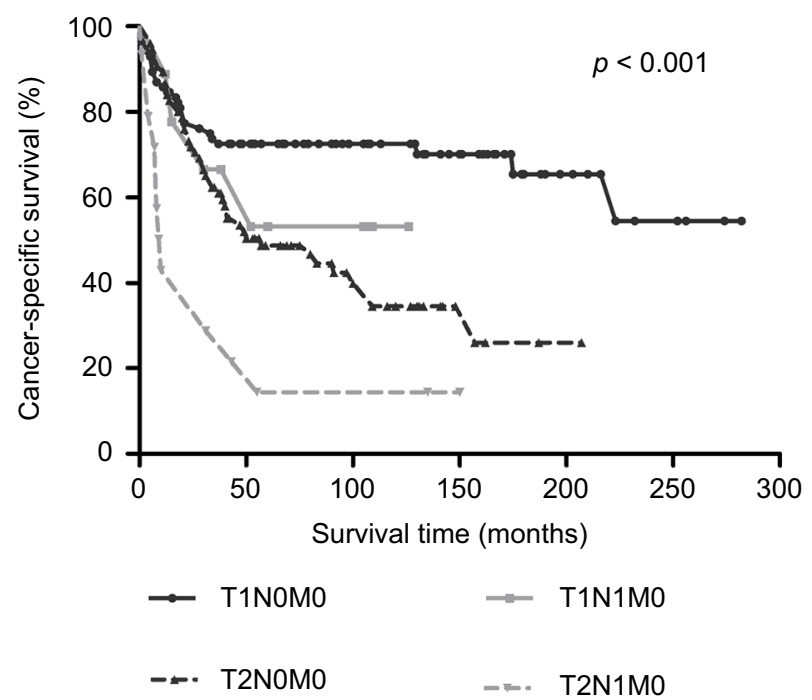

B

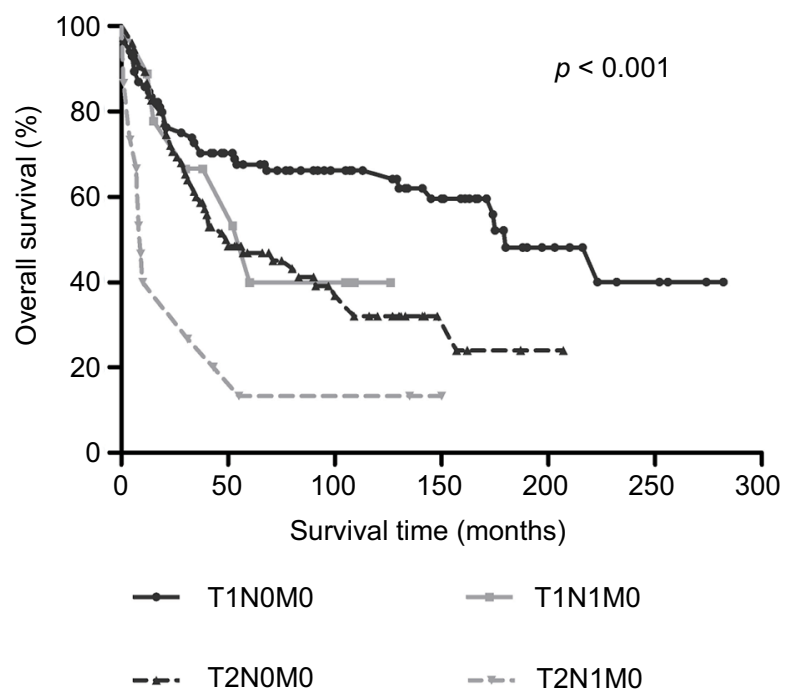

Figure 4 Kaplan-Meier survival curves according to AJCC TNM (primary tumor, regional lymph node, and distant metastasis) categories in LND (+) group. Notes: (A) Cancer-specific survival. (B) Overall survival.

Abbreviations: AJCC, American Joint Committee on Cancer; LND, lymph node dissection. 
T1N0M0. These results may be related to inadequate cases with LNM in AJCC T1. In addition, some unknown factors may have influenced these results, such as whether chemotherapy was used after surgery, chemotherapy regimens, the scope of LND, and so on.

\section{Limitations}

Several limitations exist in our study. First, it is possible that there was selection bias in the process of data screening and partial incomplete data because this is a non-randomized retrospective study. Second, there was a lack of central pathological consultation of patients registered in the SEER database. Third, the extent of debulking surgery, scope of lymphadenectomy, and the information regarding chemotherapy use were not available in SEER database while a majority of women with early stage OCS could have received optimal debulking. Finally, the survival outcomes of patients with OCS are also affected by socioeconomic status and environmental exposures.

\section{Conclusion}

Our study analyzes LNM prevalence and the role of LND and LNM on survival in early OCS (AJCC T1 and AJCC T2). Patients with LNM in AJCC T2 were shown to have poor survival outcomes and LND improved the prognosis of patients with AJCC T2. Although there was no demonstration that LND and LNM had an impact on AJCC T1 survival, patients with OCS in AJCC T1 should be considered to undergo LND owe to $9.6 \%$ LNM rate. A large-scale multi-center trial may not be feasible because of the rarity of OCS; such a study would have provided further evidence on the role of LND and LND in AJCC T1.

\section{Acknowledgment}

This study was supported by two grants: National Key R\&D Program of China (2016YFC1303704) and CAMS Innovation Fund for Medical Sciences (CIFMS; 2016-I2M-1-001).

\section{Disclosure}

The authors report no conflicts of interest in this work.

\section{References}

1. Mano MS, Rosa DD, Azambuja E, et al. Current management of ovarian carcinosarcoma. Int J Gynecol Cancer. 2007;17(2):316-324.

2. Rauh-Hain JA, Diver EJ, Clemmer JT, et al. Carcinosarcoma of the ovary compared to papillary serous ovarian carcinoma: a SEER analysis. Gynecol Oncol. 2013;131(1):46-51.

3. Rauh-Hain JA, Gonzalez R, Bregar AJ, et al. Patterns of care, predictors and outcomes of chemotherapy for ovarian carcinosarcoma: A National Cancer Database analysis. Gynecol Oncol. 2016;142(1):38-43.

4. Loizzi V, Cormio G, Camporeale A, et al. Carcinosarcoma of the ovary: analysis of 13 cases and review of the literature. Oncology. 2011;80(1-2):102-106.

5. Brown E, Stewart M, Rye T, et al. Carcinosarcoma of the ovary: 19 years of prospective data from a single center. Cancer. 2004;100(10):2148-2153.

6. Jernigan AM, Fader AN, Nutter B, et al. Ovarian carcinosarcoma: effects of cytoreductive status and platinum-based chemotherapy on survival. Obstet Gynecol Int. 2013;2013:490508.

7. Leiser AL, Chi DS, Ishill NM, Tew WP. Carcinosarcoma of the ovary treated with platinum and taxane: the memorial Sloan-Kettering Cancer Center experience. Gynecol Oncol. 2007;105(3):657-661.

8. Rauh-Hain JA, Growdon WB, Rodriguez N, et al. Carcinosarcoma of the ovary: a case-control study. Gynecol Oncol. 2011;121(3):477-481.

9. Inthasorn P, Beale P, Dalrymple C, Carter J. Malignant mixed mullerian tumour of the ovary: prognostic factor and response of adjuvant platinumbased chemotherapy. Aust N Z J Obstet Gynaecol. 2003;43(1):61-64.

10. Garg G, Shah JP, Kumar S, et al. Ovarian and uterine carcinosarcomas: a comparative analysis of prognostic variables and survival outcomes. Int J Gynecol Cancer. 2010;20(5):888-894.

11. Lu CH, Chen IH, Chen YJ, et al. Primary treatment and prognostic factors of carcinosarcoma of the ovary, fallopian tube, and peritoneum: a Taiwanese Gynecologic Oncology Group Study. Int J Gynecol Cancer. 2014;24(3):506-512.

12. Chang J, Sharpe JC, A'Hern RP, et al. Carcinosarcoma of the ovary: incidence, prognosis, treatment and survival of patients. Ann Oncol. 1995;6(8):755-758.

13. Gourley C, Al-Nafussi A, Abdulkader M, Smyth JF, Gabra H. Malignant mixed mesodermal tumours: biology and clinical aspects. Eur J Cancer. 2002;38(11):1437-1446.

14. del Carmen MG, Birrer M, Schorge JO. Carcinosarcoma of the ovary: a review of the literature. Gynecol Oncol. 2012;125(1):271-277.

15. Kunkel J, Peng Y, Tao Y, Krigman H, Cao D. Presence of a sarcomatous component outside the ovary is an adverse prognostic factor for primary ovarian malignant mixed mesodermal/mullerian tumors: a clinicopathologic study of 47 cases. Am J Surg Pathol. 2012;36(6):831-837.

16. Harris MA, Delap LM, Sengupta PS, et al. Carcinosarcoma of the ovary. Br J Cancer. 2003;88(5):654-657.

17. Silverberg SG, Major FJ, Blessing JA, et al. Carcinosarcoma (malignant mixed mesodermal tumor) of the uterus. A Gynecologic Oncology Group pathologic study of 203 cases. Int J Gynecol Pathol. 1990;9(1):1-19.

18. Berton-Rigaud D, Devouassoux-Shisheboran M, Ledermann JA, et al. Gynecologic Cancer InterGroup (GCIG) consensus review for uterine and ovarian carcinosarcoma. Int J Gynecol Cancer. 2014;24(9 Suppl 3): S55-60.

19. Sukur YE, Taskin S, Varli B, et al. Prognostic factors for disease-free and overall survival of patients with uterine carcinosarcoma. Int J Clin Oncol. 2018;23(1):114-120. 


\section{Supplementary material}

Table SI Multiple comparisons of CSS and OS in different AJCC TNM (primary tumor, regional lymph node, and distant metastasis) categories

\begin{tabular}{|c|c|c|c|c|c|c|c|c|c|}
\hline \multirow[t]{2}{*}{ Survival } & \multirow[t]{2}{*}{ Categories } & \multicolumn{2}{|c|}{ TINOMO } & \multicolumn{2}{|c|}{ TINIMO } & \multicolumn{2}{|c|}{ T2NOMO } & \multicolumn{2}{|c|}{ T2NIMO } \\
\hline & & $\chi^{2}$ & $p$ & $\chi^{2}$ & $p$ & $\chi^{2}$ & $p$ & $\chi^{2}$ & $p$ \\
\hline \multirow[t]{4}{*}{ CSS } & TINOMO & & & 0.78 & 0.377 & 12.84 & $<0.001$ & 21.48 & $<0.001$ \\
\hline & TINIMO & 0.78 & 0.377 & & & 0.48 & 0.489 & 4.49 & 0.034 \\
\hline & T2NOMO & 12.84 & $<0.001$ & 0.48 & 0.489 & & & 7.57 & 0.006 \\
\hline & T2NIMO & 21.48 & $<0.001$ & 4.49 & 0.034 & 7.57 & 0.006 & & \\
\hline \multirow[t]{4}{*}{ OS } & TINOMO & & & 1.29 & 0.257 & 10.43 & 0.001 & 20.86 & $<0.001$ \\
\hline & TINIMO & 1.29 & 0.257 & & & 0.22 & 0.642 & 4.11 & 0.043 \\
\hline & T2NOMO & 10.43 & 0.001 & 0.22 & 0.642 & & & 7.89 & 0.005 \\
\hline & T2NIMO & 20.86 & $<0.001$ & 4.11 & 0.043 & 7.89 & 0.005 & & \\
\hline
\end{tabular}

Abbreviations: CSS, cancer-specific survival; OS, overall survival; AJCC, American Joint Committee on Cancer.

\section{Publish your work in this journal}

Cancer Management and Research is an international, peer-reviewed open access journal focusing on cancer research and the optimal use of preventative and integrated treatment interventions to achieve improved outcomes, enhanced survival and quality of life for the cancer patient. The manuscript management system is completely online and includes a very quick and fair peer-review system, which is all easy to use. Visit http://www.dovepress.com/testimonials.php to read real quotes from published authors. 